Sādhanā Vol. 40, Part 6, September 2015, pp. 1955-1974. (C) Indian Academy of Sciences

\title{
Stability analysis of sandy slope considering anisotropy effect in friction angle
}

\author{
HAMED FARSHBAF AGHAJANI ${ }^{1}$, HOSSEIN SALEHZADEH ${ }^{2, *}$ \\ and HABIB SHAHNAZARI ${ }^{2}$ \\ ${ }^{1}$ Faculty of Engineering, Azarbaijan Shahid Madani University, \\ Kilometere 35 of Tabriz/Azarshahr Road, P.O. Box 53714-161, Tabriz, Iran \\ ${ }^{2}$ School of Civil Engineering, Iran University of Science and Technology, \\ P.O.B. 16765-163 Narmak, Tehran, Iran \\ e-mail: hfarshbaf@iust.ac.ir; salehzadeh@iust.ac.ir; hshahnazari@iust.ac.ir
}

MS received 16 April 2014; accepted 12 April 2015

\begin{abstract}
This paper aims to investigate the effect of anisotropy of shear strength parameter on the stability of a sandy slope by performing the limit equilibrium analysis. Because of scarcity of mathematical equation for anisotropic friction angle of sand, at first, all results of principal stress rotation tests are processed by artificial neural network and a computational procedure is developed for determining sand friction angle subjected to various loading directions. By implementing this procedure, slope stability analysis is performed in both isotropic and anisotropic conditions. The results indicate that while isotropic slope stability overestimates the factor of safety between 5 and $25 \%$ which the deviation is more for flatter slope, the location of critical slip surface is coincident in both conditions. Also in specific slip surface, the parameters of face angle, geometry of slip surface and soil properties relating to anisotropy are the main factors governing the result of anisotropic slope stability.
\end{abstract}

Keywords. Anisotropy; artificial neural network; principal stress rotation; slope stability; anisotropy ratio.

\section{Introduction}

The direction-dependency of soil mechanical and strength properties known as anisotropy is one of the most prominent phenomena in soil mechanics and occurs in many soil depositions due to sedimentation process in water. In most alluvial soils, the grains settle in a preferred orientation in water and lay on each other in such a manner that grains longitude plane is parallel to the sedimentation layer direction. This type of grain arrangement imposes a weakness in direction of bedding plane and influences the mechanical behavior of soil deposit. Therefore, the mechanical response of soil element depends not only on the magnitude of applied stress, but also on the

${ }^{*}$ For correspondence 
orientation of principal stress relative to bedding layer (Arthur \& Menzies 1972; Aghajani \& Salehzadeh 2015; Kuerbis \& Vaid 1989). If the anisotropy feature of soil is neglected and only the strength specifications of soil in vertical direction are attributed to the whole of the soil mass, the resultant design of earth structure may accompanied by some problematic issues and the factor of safety calculated for examining the stability of earth works would be unreal and even over than real state.

The alteration of principal stress orientation during loading can be found in natural and manmade slope. In figure 1, three elements are shown at various situations along an arbitrary slip surface. The orientation of sliding plane varies in these elements and hence the principal stress directions acting in elements are different. Consequently, considering the variation of loading orientation against the sedimentation bedding along the slip surface together with anisotropic nature of soil deposition, it is necessary to attribute different mechanical properties to any points along the slip surface and slope stability should be evaluated by considering the anisotropy of soil properties.

In the last decades, some researchers have focused on the studying of anisotropy role in the stability of cohesive slope by performing stability analysis with both limit equilibrium and limit analysis methods (Lo 1965; Chen et al 1975; Su \& Liao 1999; Al-Karni \& Al-Shamrani 2000; Nian et al 2008; Shogaki \& Kumagai 2008). One of the early attempts at studying the anisotropic slope stability was made by Lo (1965) which found that the effect of anisotropy is more predominant in flatter slope than steeper. Chen et al (1975) studied the relation between undrained shear strength anisotropy and slope stability by upper bound limit analysis. They found that the anisotropy is more effective in soils with lower friction angle. (Arai \& Nakagawa 1986) found that anisotropy of shear strength does not greatly influence the location of critical slip surface. (Su \& Liao 1999) proposed the chart for the ratio of anisotropic safety factor to isotropic state in cohesive slopes. Also, they recognized that critical circular slip surface does not coincide together in both isotropic and anisotropic conditions. The study of Al-Karni \& Al-Shamrani (2000) for the anisotropy effect on slope stability of $\emptyset$-c soils by method of slides agreed the conformity of critical slip surface location in both conditions of isotropy and anisotropy. Shogaki \& Kumagai (2008) proposed a new procedure for analyzing the stability of anisotropic slope, considering both inherent and stress-induced anisotropy.

Even though the objective of most investigations of the anisotropy effect on slope stability is focused on the clayey slopes, there is noticeably scarce for studying the impact of friction angle anisotropy on the stability of coarse grained slopes. According to this shortage, this paper

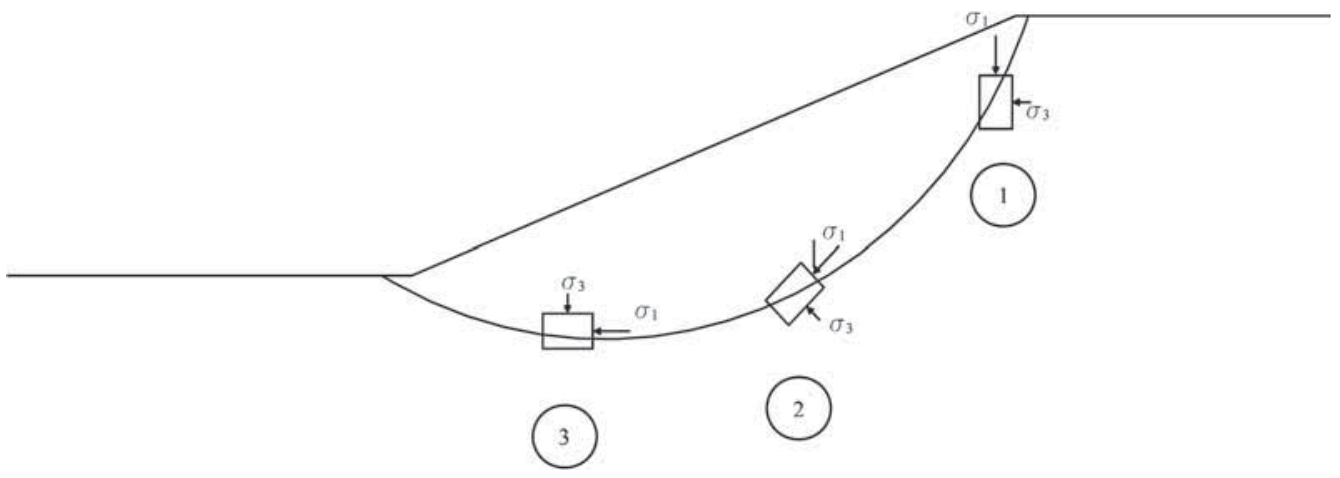

Figure 1. Illustration of stress state along a slip surface (after Uthayakumar \& Vaid (1998)). 
aims to thoroughly study the anisotropy of friction angle in sandy soils and evaluate its effect on the stability of a sandy slope by performing the limit equilibrium analysis. To this end, at first step, whole experimental data of principal stress rotation test of sands are collected and processed by means of artificial neural network (ANN). After establishing the ANN, some sensitivity analyses are performed to determine the relation between sample specifications and shear strength anisotropy. Finally, considering the variability of soil shear strength parameters with loading orientation, the stability of a sandy slope with various geometries is analyzed by the limit equilibrium method of slides.

\section{Investigation of anisotropy in sand by artificial neural network (ANN)}

Studying the role of anisotropy in response to geotechnical structure requires the constitutive model which comprehensively accounts the effect of anisotropy on the mechanical behavior of soil. Casagrande \& Carillo (1944) have presented a closed-form equation for relating between the soil cohesion in Mohr-Columb model and loading orientation that is widely implemented in stability analysis of anisotropic cohesive slope (Lo 1965; Chen et al 1975; Su \& Liao 1999; Al-Karni \& Al-Shamrani 2000; Nian et al 2008).

Despite the Casagrande and Carillo equation, unfortunately there is not any mathematical equation for friction angle parameter concerning the anisotropy effect. In other words, even though many experimental investigations have been yet conducted to study the anisotropic behavior of sands, a little attention is paid to process these test data and develop a procedure for computing the anisotropic friction angle. To overcome this shortage, in the current paper, all experimental data of anisotropy tests have been collected from the literature and statistically processed by means of the artificial neural network (ANN).

The database of anisotropy experimental data consists of principal stress rotational test results performed by using the hollow cylinder torsional shear apparatus (HCTA) (Gutierrez et al 1991; Dakoulas \& Sun 1992; Nakata et al 1998; Lade et al 2008; Tsomokos \& Georgiannou 2010). This apparatus has the ability to control the magnitude and direction of three-dimensional principal stresses within the sample (Lade 1981). At any point within the sample, the maximum principal stress makes the angle of $\alpha$ against vertical axis in the plane of sample wall. The intermediate stress is induced in radial direction and expressed by non-dimensional parameter called the intermediate principal stress ratio $(b)$ as follows (Bishop 1966)

$$
b=\frac{\sigma_{2}-\sigma_{3}}{\sigma_{1}-\sigma_{3}} .
$$

In all tests in database, the mean total principal stress, the direction of maximum principal stress and the intermediate principal stress ratio $(b)$ have been maintained constant during shearing loading and the mean total stress is set to be equal to initial consolidation confining pressure. The basic information of all 73 records of the database is presented in table 1.

Based on the findings of experimental studies, seven input variables are designated as main factors of ANN influencing the anisotropy in sands. These input factors are related to the intrinsic specifications of soil, sample condition, consolidation history and shear loading condition which include mean diameter $\left(D_{50}\right)$, grain angularity, sample void ratio $(e)$, preparation method, initial confining pressure $\left(P_{c}\right)$, the angle of maximum principal stress direction $(\alpha)$ and intermediate principal stress ratio $(b)$. The effective peak friction angle of the sample $\left(\emptyset^{\prime}\right)$ is selected as the target variable. 





Because of different ranges of input variables, all inputs are preprocessed and normalized using the following equation to fall in the bound between 0 to 1 based on the upper $\left(x_{\max }\right)$ and lower $\left(x_{\min }\right)$ value of input:

$$
X_{\text {normalized }}=\frac{x-x_{\min }}{x_{\max }-x_{\min }} .
$$

The output target variable is not subjected to normalizing and pure value is considered in the neural network. In table 2, the statistical population of input variables in three subsets of training, validation and testing together with the total set of database are presented. The participant of training, testing and validation subsets from the whole of records are 70,15 and 15\%, respectively.

The artificial neural is feed-forward back propagation type network developed by neural network toolbox in MATLAB (Demuth \& Beale 1992). The architecture of the network is composed

Table 2. The statistics of populations used for training, validation and testing of ANN.

\begin{tabular}{|c|c|c|c|c|c|c|}
\hline & $\begin{array}{c}\text { Input } \\
\text { variable }\end{array}$ & Average & $\begin{array}{l}\text { Standard } \\
\text { deviation }\end{array}$ & Maximum & Minimum & Range \\
\hline \multirow[t]{8}{*}{ Total } & Mean size of grain $\left(D_{50}\right),(\mathrm{mm})$ & 0.20 & 0.06 & 0.28 & 0.11 & 0.17 \\
\hline & Angularity ${ }^{\mathrm{a}}$ & 61.81 & 22.04 & 75.00 & 25.00 & 50.00 \\
\hline & Void ratio $(e)$ & 0.71 & 0.06 & 0.87 & 0.61 & 0.26 \\
\hline & Preparation method ${ }^{\mathrm{b}}$ & 18.47 & 4.90 & 30.00 & 10.00 & 20.00 \\
\hline & Confining pressure $\left(P_{c}\right)(\mathrm{kPa})$ & 266.31 & 278.55 & 1350.00 & 75.00 & 1275.00 \\
\hline & Angle of maximum principal stress $(\alpha)$ & $37.61^{\circ}$ & $24.61^{\circ}$ & $90^{\circ}$ & 0 & $90^{\circ}$ \\
\hline & Intermediate principal stress ratio $(b)$ & 0.41 & 0.28 & 1.00 & 0.00 & 1.00 \\
\hline & Effective peak friction angle $\left(\emptyset^{\prime}\right)$ & $39.59^{\circ}$ & $5.25^{\circ}$ & $48.90^{\circ}$ & $27.06^{\circ}$ & $21.84^{\circ}$ \\
\hline \multirow[t]{8}{*}{ Training } & Mean size of grain $\left(D_{50}\right),(\mathrm{mm})$ & 0.20 & 0.06 & 0.28 & 0.11 & 0.17 \\
\hline & Angularity ${ }^{\mathrm{a}}$ & 61.54 & 22.18 & 75.00 & 25.00 & 50.00 \\
\hline & Void ratio $(e)$ & 0.71 & 0.06 & 0.87 & 0.61 & 0.26 \\
\hline & Preparation method ${ }^{\mathrm{b}}$ & 18.85 & 5.06 & 30.00 & 10.00 & 20.00 \\
\hline & Confining pressure $\left(P_{c}\right)(\mathrm{kPa})$ & 267.58 & 287.39 & 1350.00 & 75.00 & 1275.00 \\
\hline & Angle of maximum principal stress $(\alpha)$ & $38.23^{\circ}$ & $24.73^{\circ}$ & $90^{\circ}$ & 0 & $90^{\circ}$ \\
\hline & Intermediate principal stress ratio $(b)$ & 0.43 & 0.27 & 1.00 & 0.00 & 1.00 \\
\hline & Effective peak friction angle $\left(\emptyset^{\prime}\right)$ & $39.44^{\circ}$ & $5.19^{\circ}$ & $48.90^{\circ}$ & $27.06^{\circ}$ & $21.84^{\circ}$ \\
\hline \multirow[t]{8}{*}{ Validation } & Mean size of grain $\left(D_{50}\right),(\mathrm{mm})$ & 0.19 & 0.06 & 0.25 & 0.11 & 0.14 \\
\hline & Angularity ${ }^{\mathrm{a}}$ & 60.00 & 22.91 & 75.00 & 25.00 & 50.00 \\
\hline & Void ratio $(e)$ & 0.72 & 0.06 & 0.84 & 0.67 & 0.17 \\
\hline & Preparation method ${ }^{\mathrm{b}}$ & 17.00 & 4.58 & 20.00 & 10.00 & 10.00 \\
\hline & Confining pressure $\left(P_{c}\right)(\mathrm{kPa})$ & 347.80 & 330.46 & 1000.00 & 98.00 & 902.00 \\
\hline & Angle of maximum principal stress $(\alpha)$ & $27.06^{\circ}$ & $20.94^{\circ}$ & $69.60^{\circ}$ & 0 & $69.60^{\circ}$ \\
\hline & Intermediate principal stress ratio $(b)$ & 0.35 & 0.28 & 0.90 & 0.00 & 0.90 \\
\hline & Effective peak friction angle $\left(\emptyset^{\prime}\right)$ & $38.88^{\circ}$ & $6.37^{\circ}$ & $45.40^{\circ}$ & $28.69^{\circ}$ & $16.72^{\circ}$ \\
\hline \multirow[t]{8}{*}{ Testing } & Mean size of grain $\left(D_{50}\right),(\mathrm{mm})$ & 0.18 & 0.06 & 0.25 & 0.11 & 0.14 \\
\hline & Angularity ${ }^{\mathrm{a}}$ & 65.00 & 20.00 & 75.00 & 25.00 & 50.00 \\
\hline & Void ratio $(e)$ & 0.70 & 0.05 & 0.84 & 0.61 & 0.18 \\
\hline & Preparation method ${ }^{\mathrm{b}}$ & 18.00 & 4.00 & 20.00 & 10.00 & 10.00 \\
\hline & Confining pressure $\left(P_{c}\right)(\mathrm{kPa})$ & 178.20 & 74.64 & 300.00 & 98.00 & 202.00 \\
\hline & Angle of maximum principal stress $(\alpha)$ & $44.94^{\circ}$ & $23.94^{\circ}$ & $90^{\circ}$ & $15^{\circ}$ & $75^{\circ}$ \\
\hline & Intermediate principal stress ratio $(b)$ & 0.46 & 0.25 & 1.00 & 0.10 & 0.90 \\
\hline & Effective peak friction angle $\left(\emptyset^{\prime}\right)$ & $41.09^{\circ}$ & $3.89^{\circ}$ & $47.99^{\circ}$ & $33.49^{\circ}$ & $14.50^{\circ}$ \\
\hline
\end{tabular}

a 100: angular; 75:sub-angular; 50: sub-rounded; 25: rounded.

b30: air-pluviation; 20: wet-pluviation; 10:tamping. 
of input layer with 7 neurons, output layer with one neuron and one hidden layer with 14 neurons and illustrated in figure 2. The sigmoid logistic function is used as a transfer function to calculate the output of the neurons in the hidden and output layers.

The neural network is successfully trained and validated for aforementioned subsets of principal stress rotation test records. The main criteria for checking the performance of neural network

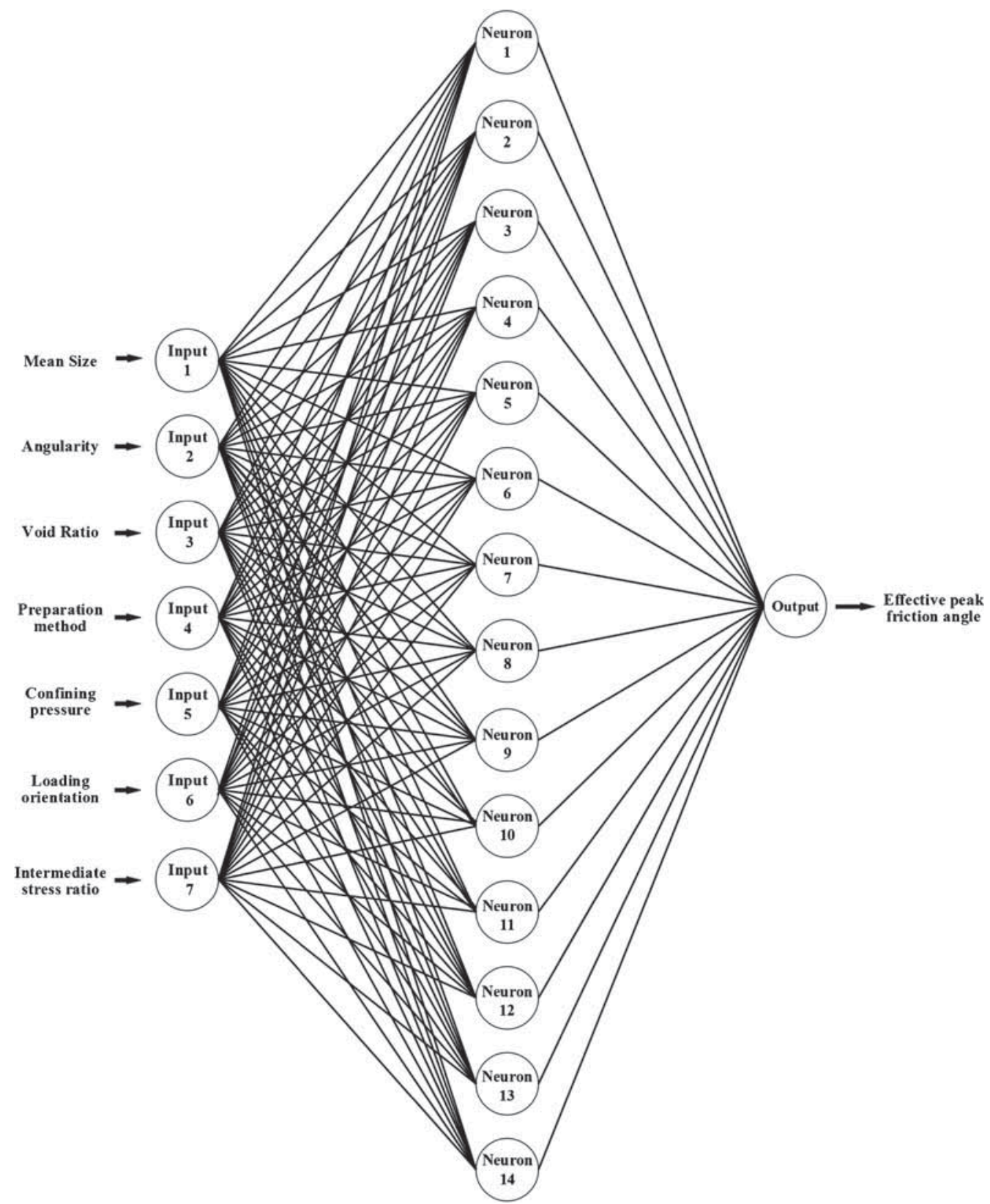

Figure 2. Architecture of neural network. 
Table 3. Analysis result of neural network.

\begin{tabular}{lccrc}
\hline & $\mathrm{r}$ & $\mathrm{r}^{2}$ & \multicolumn{1}{c}{ SSE } & RMSE \\
\hline Total Data & 0.927 & 0.859 & 218.906 & 1.768 \\
Training & 0.931 & 0.866 & 144.958 & 1.703 \\
Validation & 0.964 & 0.928 & 20.454 & 1.599 \\
Testing & 0.881 & 0.776 & 41.287 & 2.272 \\
\hline
\end{tabular}

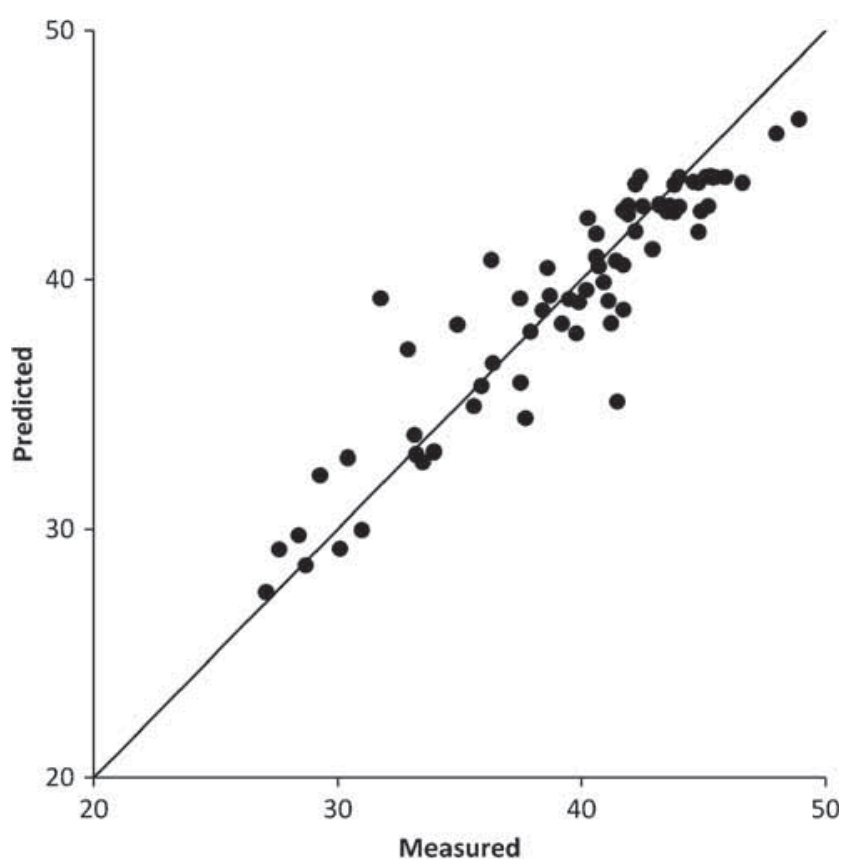

Figure 3. Measured versus predicted effective peak friction angle by neural network for all records of experimental database.

involve coefficient of correlation $(r)$, coefficient of determination $\left(r^{2}\right)$, the root mean squared error (RMSE), and the sum of squared error (SSE). The performance of network during training, validation, testing stages together with modeling of whole records are presented in table 3. As seen, the coefficient of correlation $(r)$ for fitting to the total records of the database becomes greater than 0.92 indicating the existence of a good correlation between predicted and measured value of sample friction angle. According to the Smith (1993), if the value of $|R|$ parameter for a network output becomes greater than 0.8 , strong correlation exists between two sets of variables. The result of network prediction of the whole database versus actual value of records is shown in figure 3.

\section{Sensitivity analysis of anisotropy effect}

\subsection{Effect of confining pressure}

For a sub-angular wet pluviated sand with mean diameter of $0.2 \mathrm{~mm}$ and void ratio of 0.74 , the variation of effective peak friction angle against maximum principal stress direction $(\alpha)$ in 
different confining pressures is calculated via ANN and illustrated in figure 4(b). At any level of confining pressure, with rotation of maximum principal stress direction toward the bedding angle, the shear strength of the sample is reduced. However by increasing the confining pressure, the intensity of anisotropy tends to decrease.

In figure 4(c), the graph of the friction angle versus angle of maximum principal stress is presented for looser sample having the highest void ratio among the experimental database (i.e. $e=0.87$ ). In this sample, the anisotropy effect is intensified with confining pressure and by increasing the applied confining pressure from 75 to $1350 \mathrm{kPa}$, the total loss of friction angle due
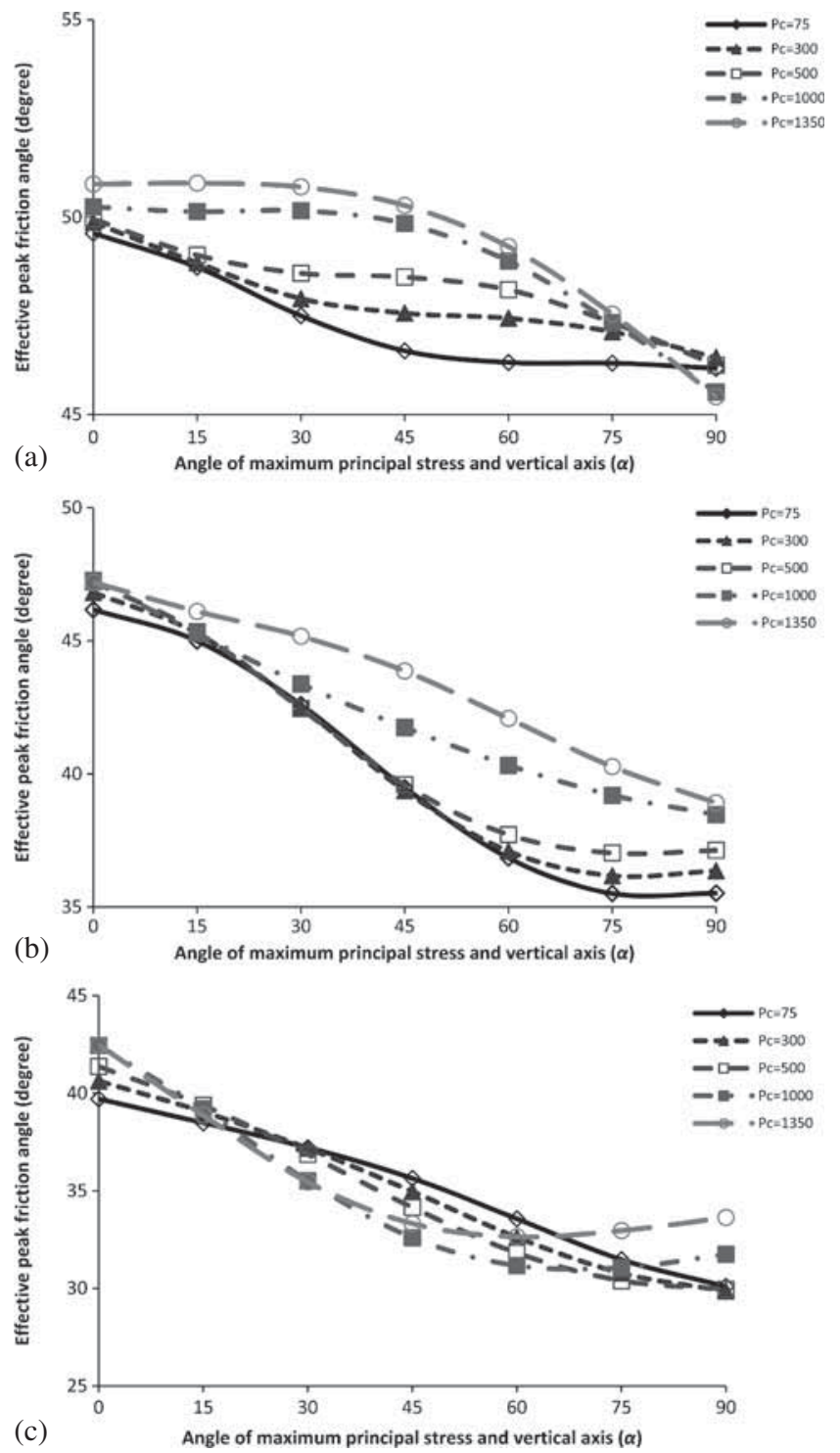

Figure 4. Effective peak friction angle versus maximum stress orientation at various levels of confining pressures in samples with sub angular grains, wet pluviated preparation method, $D_{50}=0.2 \mathrm{~mm}, \mathrm{~b}=0.5$ and having void ratio of (a) $e=0.61$, (b) $e=0.74$, (c) $e=0.87$. 
to stress rotation is amplified from 9.6 to 11.47 . In dense sample which the anisotropy effect is presented in figure 4(a), the total loss of friction angle due to anisotropy is about $3^{\circ}$ for confining pressure less than $300 \mathrm{kPa}$. Then, by increasing the applied pressures in sample, the total loss of

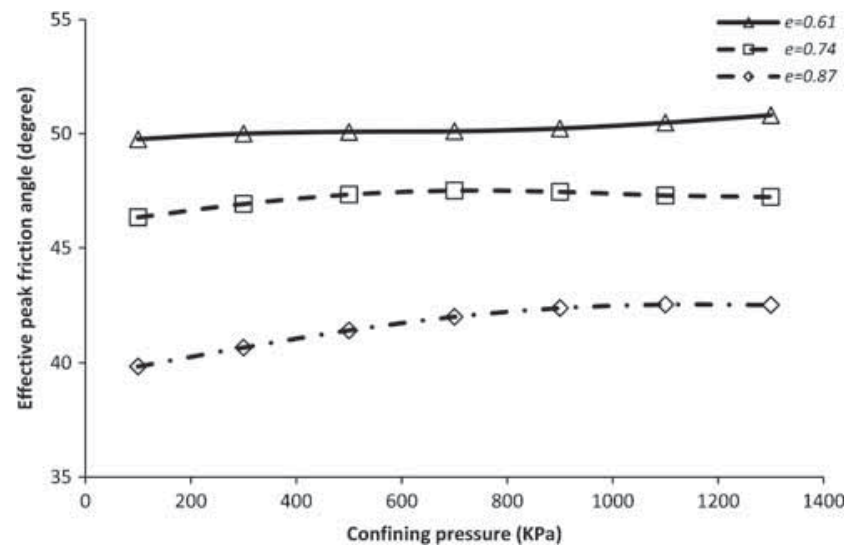

Figure 5. Effect of confining pressure on the friction angle in samples with various void ratios and subjected to vertical maximum principal stress $\left(\alpha=0^{\circ}\right)$. All samples have sub angular grains with $D_{50}=0.2$ $\mathrm{mm}, \mathrm{b}=0.5$ and wet pluviated preparation method.
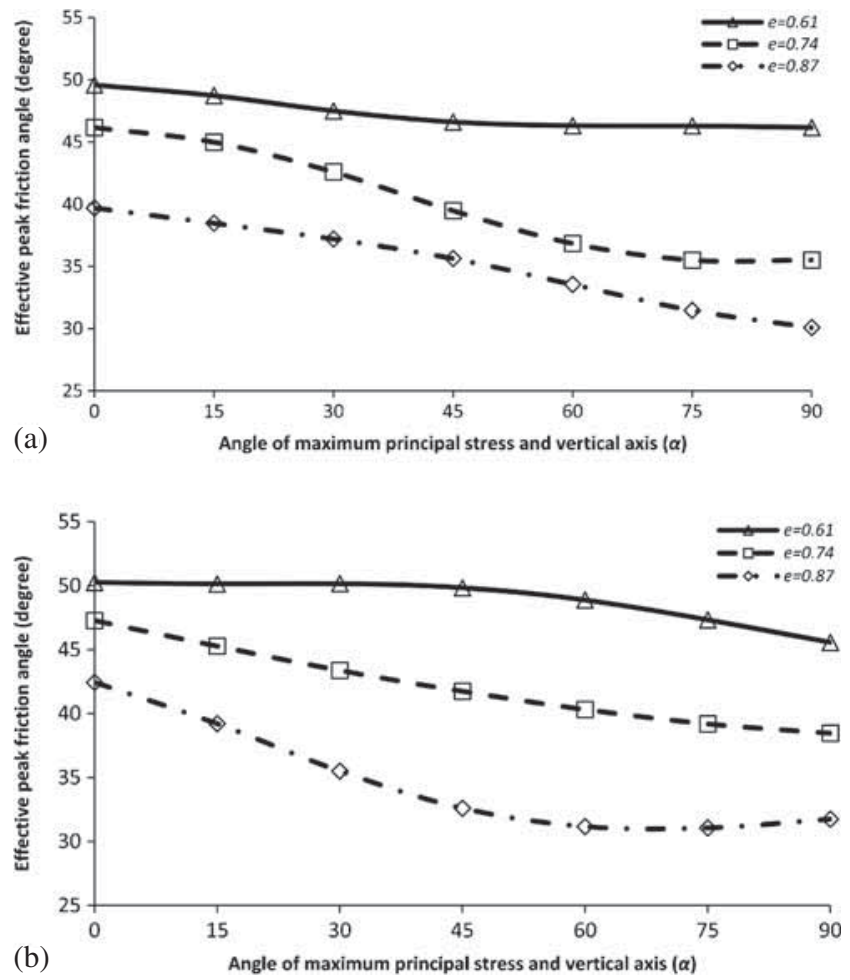

Figure 6. Effective peak friction angle versus maximum stress orientation in samples with various void ratios and having sub angular grains, wet pluviated preparation method, $D_{50}=0.2 \mathrm{~mm}, b=0.5$ and subjected to ; (a) $P_{c}=75 \mathrm{kPa}$; (b) $P_{c}=500 \mathrm{kPa}$. 
sample friction angle is increased to $5^{\circ}$. In figure 5 , the effect of confining pressure on the friction angle of samples with various void ratios and subjected to vertical loading $\left(\alpha=0^{\circ}\right)$ is presented.

\subsection{Effect of sample density}

For aforementioned sample consolidated under confining pressure of $75 \mathrm{kPa}$, the anisotropic variation of effective peak friction angle is determined at various void ratios and presented in figure 6(a). The void ratio of samples is selected based on the maximum, minimum and average of test records in experimental database.

The analysis result shows that at any loading direction, increasing void ratio causes the sample friction angle to decrease. Also, the amount of shear strength reduction due to anisotropy is completely influenced by sample density. In the dense sample with minimum void ratio, the total reduction of the sample friction angle due to stress rotation from $\alpha=0$ to $\alpha=90^{\circ}$ is only $3.4^{\circ}$. Also, in medium dense and loose sample the total reduction of friction angle due to stress rotation is about $10.6^{\circ}$ and $9.6^{\circ}$, respectively.

The graph of friction angle loss in sample with various void ratios and subjected to confining pressure of $500 \mathrm{kPa}$ is presented in figure 6(b). At this level of confining pressure, the total reduction of the soil friction angle in samples with a void ratio of $0.61,0.74$ and 0.87 are $3.7^{\circ}, 10.08^{\circ}$ and $11.4^{\circ}$, respectively. More details on the sensitivity analysis of anisotropy phenomenon can be found in other publications (Aghajani et al 2015).

\section{Slope stability analysis considering anisotropy}

By implementing the proposed computational procedure for anisotropic effective peak friction angle, the effect of anisotropy of sand shear strength on the stability of slopes can be thoroughly investigated. For this end, slopes with different geometry are considered and the stability of slopes is evaluated by supposing both isotropy and anisotropy conditions for shear strength parameters and the results of two types of stability analysis are compared to recognize the effect of anisotropy.

\subsection{Analysis method}

For each slope with specific geometry, the factor of safety is calculated using the Simplified Bishop method of limit equilibrium (Bishop 1955). The key feature of the Simplified Bishop method is that the forces between slices are assumed horizontal. The evaluations show that there is a good agreement between the factor of safety obtained by the Simplified Bishop method and other limit equilibrium procedures that fully satisfy static equilibrium (Bishop 1955; Wright et al 1973; Fredlund \& Krahn 1977; Duncan \& Wright 1980). Also, according to findings of Wright et al (1973), the factor of safety calculated by the Simplified Bishop Procedure has favorable agreement (within about 5\%) with the factor of safety calculated using stresses computed independently using finite element procedures.

In the Simplified Bishop method, by satisfying the force equilibrium in all slices of a circular slip surface, the factor of safety (F.S.) for this slip surface can be determined from the following equation:

$$
F . S .=\frac{\sum_{i=1}^{n}\left[\frac{c^{\prime} \cdot \Delta l_{i} \cdot \cos \omega_{i}+\left(W_{i}-u_{i} \cdot \Delta l_{i} \cdot \cos \omega_{i}\right) \tan \phi_{i}^{\prime}}{\cos \omega_{i}+\left(\sin \omega_{i} \cdot \tan \phi_{i}^{\prime}\right) / F \cdot S .}\right]}{\sum_{i=1}^{n} W_{i} \cdot \sin \omega_{i}},
$$




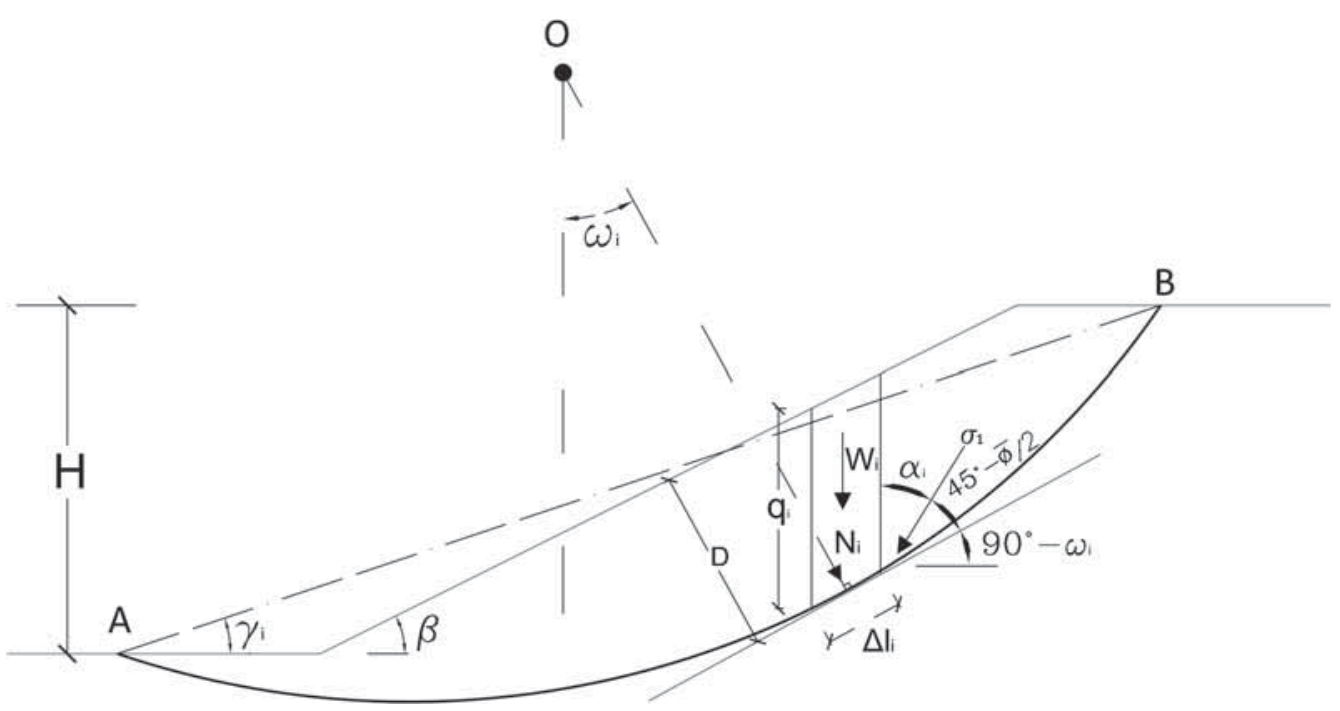

Figure 7. Geometrical consideration of slope.

where $\Delta l_{i}, W_{i}$ and $\omega_{i}$ are the width of slice bottom, weight of a slice and inclination angle of slice bottom in $i^{\text {th }}$ slice respectively which are shown in figure 7 . Also, $\phi_{i}^{\prime}, C_{i}^{\prime}$ and $u_{i}$ are effective friction angle, effective cohesion and pore water pressure of the slice, respectively. Each slip surface is divided into $n$ slices.

For avoiding the divergence of solution and false convergence in solving the factor of safety problem, the proposed approach of Chowdhury \& Zhang (1990) is employed for initial trial value of F.S.

\subsection{Slope geometry and soil parameters}

The geometry of slope subjected to stability analysis is presented in figure 7 and involved an inclined slope face limited between two horizontal surfaces. Various geometrical configurations are established for this slope by combining several magnitudes for slope height $(H=10,15,20$ and $25 \mathrm{~m}$ ) and slope face angle (denoted by $\beta$ in figure 7 and including $14^{\circ}, 21.8^{\circ}, 26.6^{\circ}, 35^{\circ}$, $45^{\circ}$ and $56.3^{\circ}$ ).

In all stability analyses in the current paper, two different conditions are supposed for shear strength of soil. In the first type of analysis, the isotropic and identical friction angle of soil layer is deemed for any point within the slope. The second type of stability analysis is performed by considering the dependency of soil shear strength with the loading direction and the friction angle for any slice in desired slip is calculated by employing the ANN.

The initial specification of slope material that is required as input variable in ANN is selected in such that lies within the range of data used in experimental database. In current research, the slope is composed of one-layered water deposited homogenous sand with $e=0.74$ and has sub-angular grains with mean diameter of $0.2 \mathrm{~mm}$ and specific gravity of 2.65. The confining stress at the bottom of each slice is assumed identical to the normal stress which arises from the projection of slice weight force acting in perpendicular direction on slice bottom and shown by $N_{i}$ in figure 7 . For determination of maximum 
principal stress direction $\left(\alpha_{i}\right)$ in $i^{\text {th }}$ slice the following equation is employed proposed by Su \& Liao (1999):

$$
\alpha_{i}=\left(\omega_{i}\right)-\left(45^{\circ}-\frac{\phi^{\prime}}{2}\right)
$$

where $\left(\omega_{i}\right)$ and $\left(\phi^{\prime}\right)$ are the angle of slice bottom against the vertical axis and soil friction, respectively. In this equation, it is assumed that the bottoms of each slice are coincident with failure plane which is occurred due to forcing of maximum principal stress. Also, it is assumed that the pattern of failure plane is independent of loading direction and isotropic friction angle is substituted in Eq. (4).

The friction angle in isotropic condition $\left(\bar{\phi}^{\prime}\right)$ is obtained from the neural network by supposing vertical loading direction $(\alpha=0)$ and then, averaging the consequent friction angle for a wide range of confining pressures. A small cohesion of $5 \mathrm{kPa}$ is supposed for shear strength of slope material.

\subsection{Stability analysis result}

For each analysis case with specified geometrical configuration for slope, a wide range of slip surfaces are considered by establishing a grid of slip rotation center together with various ranges of rotation radiuses and for each slip surface, factor of safety is calculated in both anisotropic and isotropic conditions. Then, among whole possible slip surfaces, the slip with minimum F.S. is selected as most critical slip. The value of F.S. in critical slip surface for all analysis cases in both shear strength conditions together with the anisotropy ratio are presented in table 4 . The parameter of anisotropy ratio is defined as the ratio of F.S. in anisotropic to isotropic condition and employed as an indicator for evaluating the anisotropy effect on the stability of slopes. The anisotropy ratio close to one implies less influence of anisotropy on slope stability.

As expected, by increasing the height and face angle of slope, the F.S. in both anisotropic and isotropic conditions is reduced. The anisotropy ratio completely depends on the face angle and in all analysis cases, is less than one which means that assuming isotropic condition overestimates the safety of the slope. In other words, if the anisotropy effect on shear strength of the material is neglected and all properties of material are considered as isotropic, the intended factor of safety is overestimated by an error between $5 \%$ and $25 \%$.

By comparing the anisotropy ratio for various geometrical configurations, it can be seen that in slopes with identical height, by increasing the slope face angle, the anisotropy ratio is increased and approached to one. Generally, the range of the anisotropy ratio varies from 0.75 for flatter slope to about 0.92 for steeper slope.

Survey of critical slip surface location in both anisotropic and isotropic conditions infers that the location of critical slip is not generally influenced by anisotropy and in most analysis cases, the critical slip surfaces in both conditions become identical. For instance, the critical slip surfaces in three analysis cases of (1) $H=10 \mathrm{~m}$ and $\beta=14^{\circ}$, (2) $H=10 \mathrm{~m}$ and $\beta=56.3^{\circ}$ and (3) $H=25 \mathrm{~m}$ and $\beta=14^{\circ}$ are same in both isotropic and anisotropic conditions which are shown in figure 8(a), 8(b) and 8(c), respectively. In other cases such a slope with a height of 25 and face angle of $56.3^{\circ}$ shown in figure 8(d), the critical slip surface in anisotropic condition is located in closely deeper situation relative to isotropic slip. The conformity of the critical slip surface location in anisotropic cohesive slopes was previously observed by Arai \& Nakagawa (1986) and Al-Karni \& Al-Shamrani (2000).

The other main consequent is that in slopes with same height, the depth of the critical slip surface (equal to the maximum distance of slope face from slip surface in perpendicular direction 


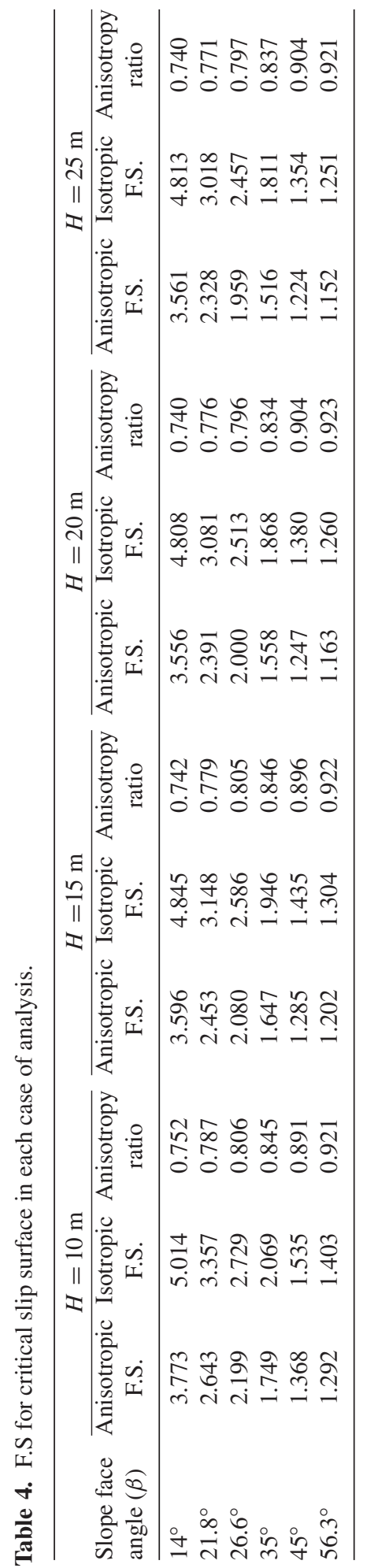




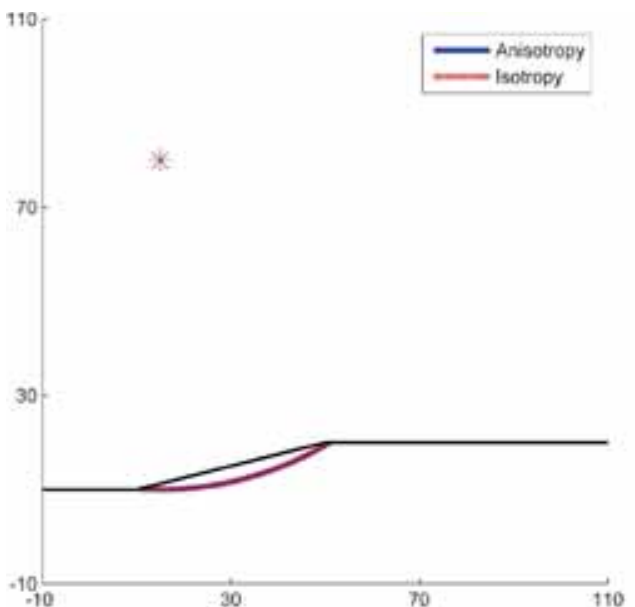

(a)

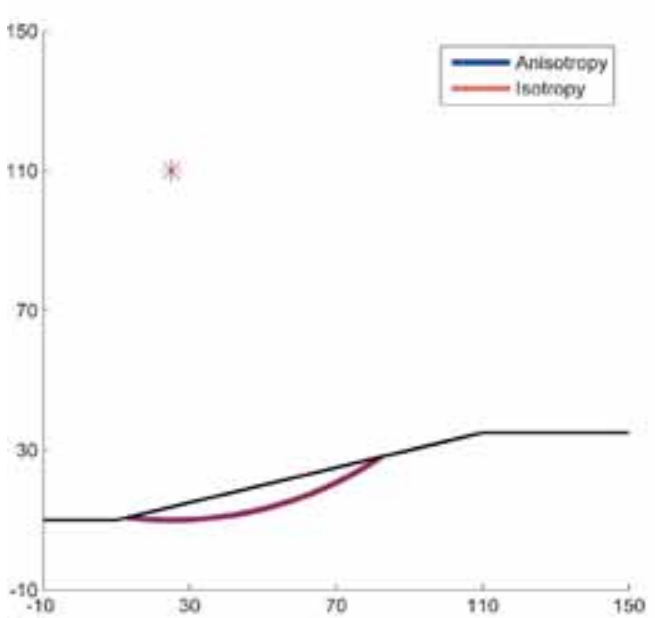

(c)

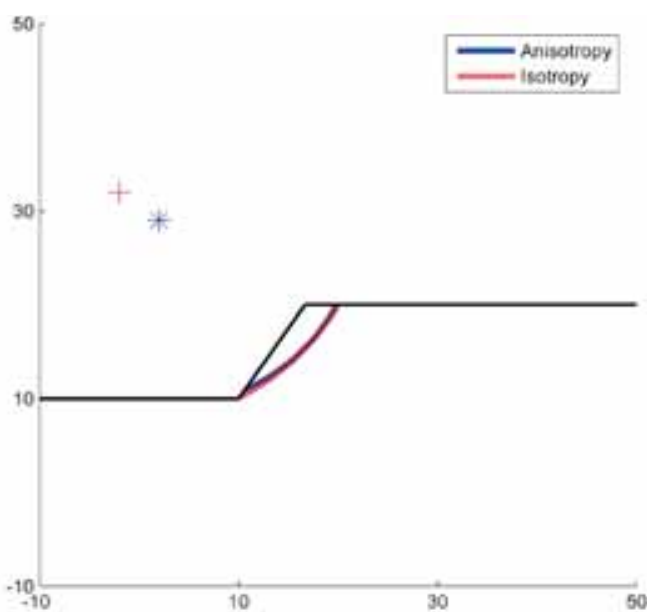

(b)

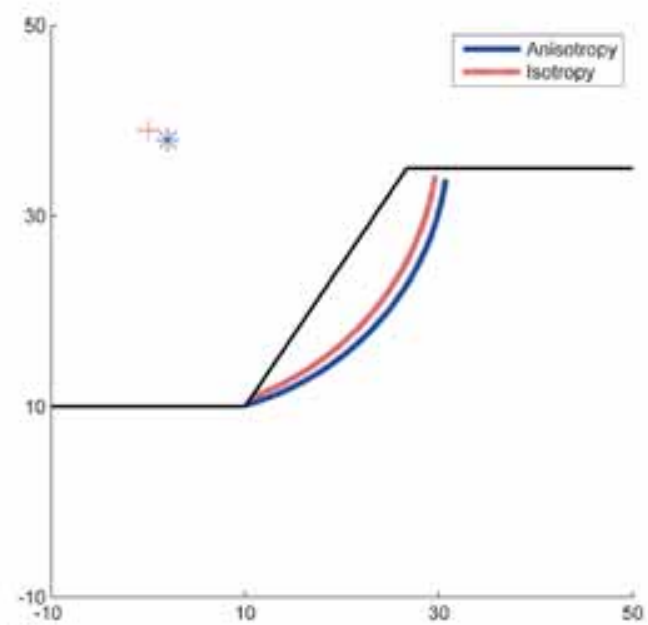

(d)

Figure 8. Location of critical slip in slopes: (a) $H=10 \mathrm{~m}$ and $\beta=14^{\circ}$, (b) $H=10 \mathrm{~m}$ and $\beta=56.3^{\circ}$, (c) $H=25 \mathrm{~m}$ and $\beta=14$ and (d) $H=25 \mathrm{~m}$ and $\beta=56.3^{\circ}$.

to slope face denoted by $D$ in figure 7) in both isotropic and anisotropic conditions are decreased by increasing the slope face inclination angle. In slope with $H=25 \mathrm{~m}$, increasing the face angle from $14^{\circ}$ to $56.3^{\circ}$ causes the depth of critical slip surfaces in anisotropic condition to decrease from $6.62 \mathrm{~m}$ to $4.93 \mathrm{~m}$.

\section{Factors enhancing the anisotropy in slope}

For determining which geometrical property of slip surface or slope is playing as a dominant factor to increase the anisotropy effect, in slope with $H=20 \mathrm{~m}$ and $\beta=14^{\circ}$, various slip surfaces with different depths (including 3, 7, 15 and $20 \mathrm{~m}$ ) and lengths (which measured on the slope face and denoted by $L$ in figure 7 ) are selected and stability of each slip surface is analyzed in 


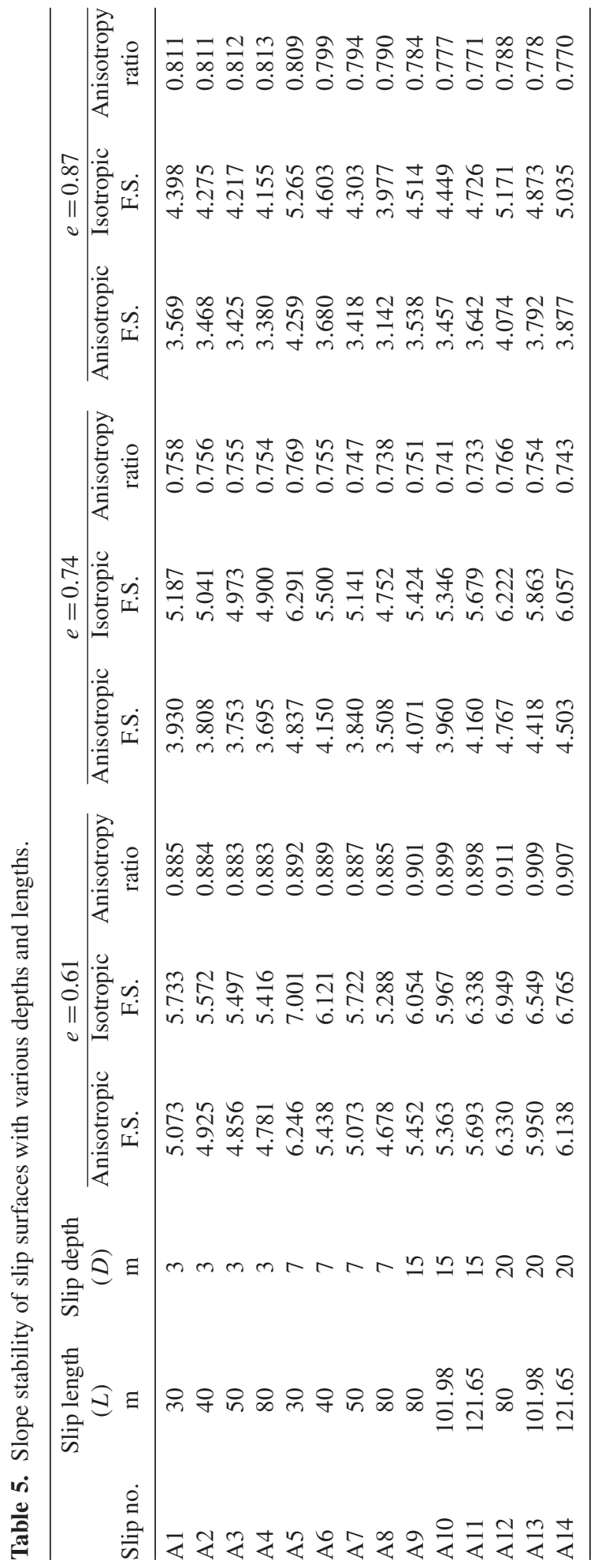


both isotropic and anisotropic conditions. By combining these values for depth and slip length, 14 various but similar categorized slip surfaces are emerged which the geometry specifications are presented in table 5 .

Moreover, in order to clarify the effect of slope material on the enhancing the anisotropy effect, the stability analysis are performed for all aforementioned slip surfaces by considering three different void ratios including $0.61,0.74$ and 0.87 which are supposed based on data of experimental database. The anisotropic friction angle for each material is calculated via ANN by supposing same input variables of sample with $e=0.74$ and presented in figure 4 . In order to avoid the more complexity, the dry density and hence the weight of soil column are assumed to be identical to soil density with void ratio of 0.74 .

The analysis results including the factor of safety for isotropic and anisotropic conditions together with the anisotropy ratio are presented in table 5 for all 14 slip surfaces. By comparing the F.S. in group of slips surfaces with identical depth $(D)$ and different lengths $(L)$, it can be seen that by increasing the slip length, the factor of safety in both conditions is gradually decreased. However in spite of the wide range of slip length in each slip group, the variation of anisotropy ratio is minor. Therefore, one can claim that the slip surface length cannot be accounted as a key factor governing the anisotropy effect on slope stability.

In contrast to slip length, the variation of the anisotropy ratio with slip depth is obvious. In a group containing slip numbers of A4, A8, A9 and A12 having identical length of $80 \mathrm{~m}$ and different depths, the anisotropy ratio is presented in figure 9 for three levels of void ratio. As seen, the anisotropy ratio is completely associated with the slip depth and may be increased or decreased depending on the void ratio of the material.

In dense material, increasing the depth of slip surfaces causes the anisotropy ratio to increase. It means that in densest material the anisotropy effect is more profound in shallower slip surfaces and by increasing the depth of the slip, the difference between isotropic and anisotropic F.S. tends to decrease. However in soil with medium void ratio, this trend can be found with slight intensity. Regarding to the graph of figure 9, by increasing the depth of slip surface up to $7 \mathrm{~m}$, the anisotropy ratio is reduced. Then by increasing the slip depth from $7 \mathrm{~m}$ to $20 \mathrm{~m}$, the anisotropy ratio rises from 0.738 to 0.766 . Unlike the dense and medium dense materials, the descending trend is governed on the variation of the anisotropy ratio in the loose slope (with $e=0.87$ ). In this slope, by increasing the depth of slip surface, the effect of anisotropy on the slope stability is intensified and the amount of the anisotropy ratio is gradually reduced.

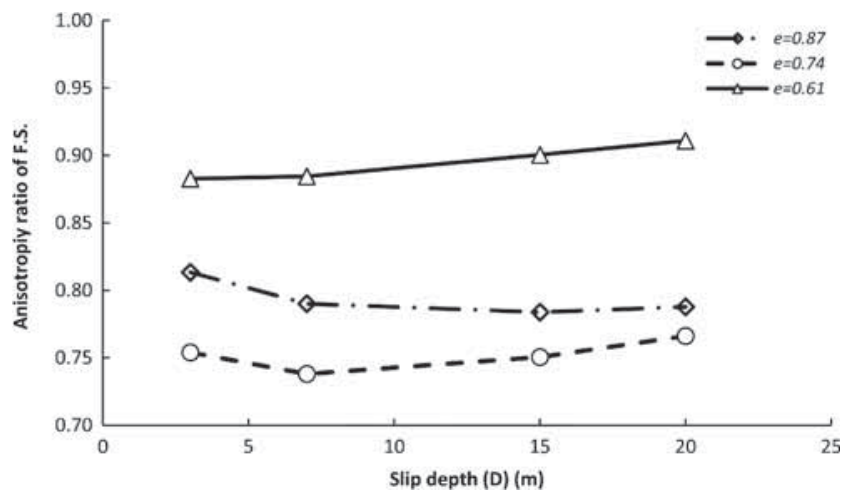

Figure 9. Graph of anisotropiy ratio of F.S. for slips with differnt depth and identical length embeded in slope with $H=20 \mathrm{~m}$ and $\beta=14^{\circ}$. 
The main reason for enhancing the anisotropy effect in shallower slip surface in dense and medium dense slops seems that by decreasing the depth of slip surface, the weight of soil column in slices comprising the slip is reduced. This leads to decrease the confining pressure acting on the base of slices. As mentioned in sensitivity analysis of the anisotropy phenomenon, increasing of confining pressure cause to reduce the friction angle loss due to stress rotation in medium dense and dense samples. Therefore, the depth of the slip surface directly influences the anisotropic friction angle in each slice and hence plays a significant role in the anisotropic analysis of slope stability.

For clarifying the effect of soil porosity on the anisotropic slope stability from other factors, anisotropy ratio is compared to a particular slip comprised of different materials. Regarding to graph of figure 9 , in slices with identical depth, the greatest value of anisotropy ratio belongs to dense soil with $e=0.61$ and the anisotropy ratio for all slips becomes greater than 0.88 . According to the graph of figure 6, the anisotropy effect in shear strength becomes minimum in dense sample.

One remarkable note about the graph of figure 9 is that in slips with equal depth, the anisotropy ratio for looser slope becomes greater than medium dense slope. For explaining this unexpected trend, one should refer to the manner of anisotropic behavior of sand at lower confining pressure. As mentioned in sensitivity analysis, at confining pressure lower than $75 \mathrm{kPa}$, the total reduction of the peak friction angle due to stress rotation in looser sample is somewhat less than medium dense sample.

For investigation the effect of slope geometry on the excitation of the anisotropy, another set of analyses is performed for two shallow (with $D=3 \mathrm{~m}$ ) and deep (with $D=7 \mathrm{~m}$ ) slips embedded in slope with various face angles and constant height of $20 \mathrm{~m}$ that comprising of medium dense material. The analysis result including isotropic and anisotropic factor of safety together with the anisotropy ratio are presented in table 6 . The interesting matter is that in both shallow and deep slip surfaces, by the increasing steepness of slope face, the anisotropy ratio tends to increase. This finding implies that in slope with a greater face gradient, the anisotropy effect on the slope stability becomes less significant. The main reason for this trend is that by increasing the face gradient, the angle of slice bottom $\left(\omega_{i}\right.$ in figure 7$)$ is increased and according to Eq. (4) cause to decrease the angle of maximum principal stress in each slice and consequently, the anisotropy effect reduces.

Table 6. Slope stability of specified slip surfaces with various slope face gradient.

\begin{tabular}{|c|c|c|c|c|c|c|c|}
\hline $\begin{array}{l}\text { Slip } \\
\text { no. }\end{array}$ & $\begin{array}{c}\begin{array}{c}\text { Slip } \\
\text { length }(L)\end{array} \\
\frac{\mathrm{m}}{}\end{array}$ & $\begin{array}{c}\begin{array}{c}\text { Slip } \\
\text { depth }(D)\end{array} \\
\mathrm{m}\end{array}$ & $\begin{array}{c}\text { Slope face } \\
\text { angle }(\beta)\end{array}$ & $\begin{array}{c}\begin{array}{c}\text { Slope } \\
\text { height }(H)\end{array} \\
\frac{\mathrm{m}}{}\end{array}$ & $\begin{array}{c}\text { Anisotropic } \\
\text { F.S. }\end{array}$ & $\begin{array}{c}\text { Isotropic } \\
\text { F.S. }\end{array}$ & $\begin{array}{l}\text { Anisotropic } \\
\text { ratio }\end{array}$ \\
\hline B1 & 20 & 3 & $14^{\circ}$ & 20 & 4.301 & 5.615 & 0.766 \\
\hline B2 & 20 & 3 & $21.8^{\circ}$ & 20 & 2.794 & 3.556 & 0.786 \\
\hline B3 & 20 & 3 & $26.6^{\circ}$ & 20 & 2.304 & 2.879 & 0.800 \\
\hline B4 & 20 & 3 & $35^{\circ}$ & 20 & 1.754 & 2.116 & 0.829 \\
\hline B5 & 20 & 3 & $45^{\circ}$ & 20 & 1.347 & 1.552 & 0.868 \\
\hline B6 & 30 & 7 & $14^{\circ}$ & 20 & 4.473 & 5.863 & 0.763 \\
\hline B7 & 30 & 7 & $21.8^{\circ}$ & 20 & 2.880 & 3.714 & 0.775 \\
\hline B8 & 30 & 7 & $26.6^{\circ}$ & 20 & 2.378 & 3.030 & 0.785 \\
\hline B9 & 30 & 7 & $35^{\circ}$ & 20 & 1.790 & 2.217 & 0.807 \\
\hline B10 & 30 & 7 & $45^{\circ}$ & 20 & 1.407 & 1.688 & 0.834 \\
\hline
\end{tabular}




\section{Design remarks}

Regarding to the results of aforementioned investigations, it can be said that issues relating to the geometry of slope, geometry of slip surface and isotropic soil shear strength properties are factors playing a main role in influencing the result of anisotropic slope stability.

Given the above-mentioned factors affecting anisotropy ratio, the value of this parameter in each slip is normalized by dividing by the tangent of isotropic friction angle $\left(\bar{\phi}^{\prime}\right)$, tangent of average slip inclination angle and maximum height of slip as following and a new parameter is emerged which called the normalized anisotropy ratio of F.S.:

$$
\text { normalized anisotropy ratio of F.S. }=\frac{\left(F \cdot S \cdot \text { anisotropy } / F . S \cdot \text { isotropy }_{\text {s }}\right)}{\tan \bar{\phi}^{\prime} \cdot \tan \gamma_{i} \cdot q_{\max }} \text {. }
$$

The average inclination angle of slip is the angle between horizontal axis and a line connecting the entry point of slip to the exit point on the slope face and designated in figure 7 by $\gamma_{i}$. Also, the maximum height of slip $\left(q_{\max }\right)$ is defined as the maximum value of vertical thickness $\left(q_{i}\right)$ among the slices of slip (c.f. figure 7).

The normalized anisotropy ratio of F.S. parameter is calculated for whole possible slip surfaces in all slopes with various geometries and composed of three types of material. The graph of this normalized parameter against the maximum height of slips is presented in figure 10. This graph embraces the normalized anisotropy ratio of F.S. for almost 6,000 slip surfaces and hence gives a comprehensive and appropriate chart for determining the ratio of anisotropic factor of safety.

It should be noted that despite the fact that neglecting the anisotropy effect on slope stability to overestimates the factor of safety, the most of commercial softwares for slope stability analysis has not the ability of anisotropic stability analysis and if any, it requires importing the appropriate function for relating shear strength parameters to the loading orientation. Furthermore, the possibility of utilizing the proposed ANN for accounting the anisotropy of peak friction angle in sands in conventional software is questionable or associated with difficulty.

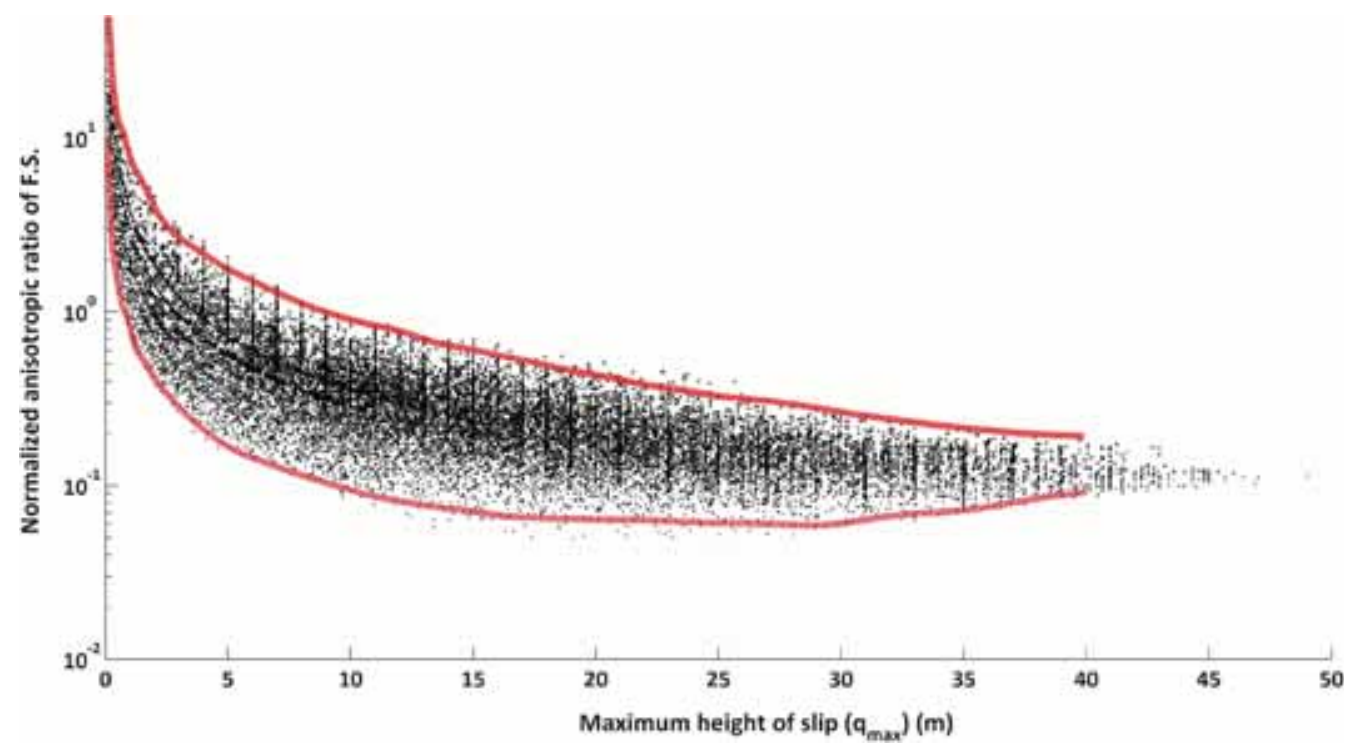

Figure 10. Normalized anisotropiy ratio of F.S. versus maximum hieght of slip. 
Therefore, according to convenience of calculating isotropic factor of safety by available software and the conformity of critical slip surface in both conditions, for accounting the anisotropy effect, it is sufficient to multiply derived factor of safety from isotropic slope stability in a reduced coefficient of the anisotropy ratio which can be easily determined for any slip surfaces with arbitrary geometry by using the graph of figure 10 .

\section{Conclusion}

In this paper, the variation of shear strength in sand due to anisotropy is modeled by processing current experimental data of anisotropy tests via artificial neural network. Then by implementation of anisotropy modeling and performing the limit equilibrium analysis, the effect of friction angle anisotropy in the stability of a sandy slope subjected to various geometrical configurations is investigated. The outcomes of analyses can be summarized as follows:

(i) Seven factors are introduced as input variables for determination of sample effective peak friction angle in artificial neural network. The orientation of loading has more contribution and relative importance in sample friction angle.

(ii) The anisotropy effect is minor in dense sample. At lower confining pressure, the total loss of sample friction angle becomes slightly more in loose sample than medium dense. But by increasing the confining pressure, the amount of shear strength reduction increases by increasing of sample porosity.

(iii) Even though in medium dense and dense sample increasing the confining pressure cause the anisotropy effect to reduce, in loose sample the anisotropy effect becomes greater with increasing the confining pressure.

(iv) According to the slope stability analysis, the anisotropy effect causes the factor of safety for critical slip surface deviates from isotropic condition and supposing isotropic condition for shear strength parameters of slope material overestimates the factor of safety between 5 and $25 \%$. This deviation is more found in flatter slope rather than steeper. However, the critical slip surface are identical in both isotropic and anisotropic conditions.

(v) Geometry of slope, geometry of slip surface and soil shear strength properties are the main factors influencing the result of anisotropic slope stability. By increasing the face angle, the anisotropic factor of safety approaches toward the isotropic. Also, in individual slip, by increasing the depth of slip surface, the anisotropy ratio is reduced. Among the physical and mechanical specifications of slope material, the properties which are provided as input variables for modeling of anisotropy in artificial neural network (such as void ratio) are directly related to the anisotropy ratio.

(vi) Regarding to the coincidence of critical slip surface in both anisotropic and isotropic conditions, for accounting the anisotropy effect on slope stability, it is sufficient to modify the resultant critical factor of safety from conventional isotropic slope stability by a decreasing coefficient called the normalized anisotropy ratio of F.S.. In this paper, a chart is presented for this parameter based on the depth of the critical slip.

\section{References}

Aghajani H F and Salehzadeh H 2015 Anisotropic behavior of the Bushehr carbonate sand in the Persian Gulf. Arabian J. Geosci. 8(10): 8197-8217. doi: 10.1007/s12517-014-1758-3 
Aghajani H F, Salehzadeh H and Shahnazari H 2015 Application of artificial neural network for calculating anisotropic friction angle of sands and effect on slope stability. J. Central South University 22(5): 18781891. doi: 10.1007/s11771-015-2707-3

Al-Karni A A and Al-Shamrani M A 2000 Study of the effect of soil anisotropy on slope stability using method of slices. Comput. Geotech. 26(2): 83-103

Arai K and Nakagawa M 1986 Influence of strength anisotropy on the search for critical noncircular slip surface. Soils Foundations 26(3): 129-136

Arthur J R F and Menzies B K 1972 Inherent anisotropy in a sand. Geotechnique 22(1): 115-129

Bishop A W 1955 The use of the Slip Circle in the stability analysis of slopes. Géotechnique 5(1): 7-17

Bishop A W 1966 The strength of soils as engineering materials. Rankine Lecture Geotechnique 16(2): 91130

Casagrande A and Carillo N 1944 Shear failure of anisotropic materials. J. Boston Soc. Civil Eng. 31(4): $74-87$

Chen W-F, Snitbhan N and Fang H-Y 1975 Stability of slopes in anisotropic, nonhomogeneous soils. Canadian Geotech. J. 12(1): 146-152

Chowdhury R and Zhang S 1990 Convergence aspect of limit equilibrium methods for slopes. Canadian Geotech. J. 27(1): 145-151

Dakoulas P and Sun Y 1992 Fine Ottawa sand: Experimental behavior and theoretical predictions. $J$. Geotech. Eng. 118(12): 1906-1923

Demuth H and Beale M 1992 MATLAB: Neural network toolbox: User's guide [EB/OL]. MathWorks, Incorporated. www.mathworks.com

Duncan J and Wright S 1980 The accuracy of equilibrium methods of slope stability analysis. Eng. Geol. 16(1): $5-17$

Fredlund D and Krahn J 1977 Comparison of slope stability methods of analysis. Canadian Geotech. J 14(3): 429-439

Gutierrez M, Ishihara K and Towhata I 1991 Flow theory for sand during rotation of principal stress direction. Soils Foundations 31(4): 121-132

Kuerbis R H and Vaid Y P 1989 Undrained behaviour of clean and silty sands. Paper presented at the The 12th International Conference on Soil Mechanics and Foundation Engineering, Session 27, Rio De Janeiro, Aug. 13.18

Lade P 1981 Torsion shear apparatus for soil testing. Laboratory shear strength of soil. ASTM STP 740: $145-163$

Lade P V, Nam J and Hong W P 2008 Shear banding and cross-anisotropic behavior observed in laboratory sand tests with stress rotation. Canadian Geotech. J. 45(1): 74-84

Lo K 1965 Stability of slopes in anistropic soils. J. Soil Mech. Foundations Div 91 (ASCE\# 4405 Proceeding)

Nakata Y, Hyodo M, Murata H and Yasufuku N 1998 Flow deformation of sands subjected to principal stress rotation. Soils Foundations 38(2): 115-128

Nian T, Chen G, Luan M, Yang Q and Zheng D 2008 Limit analysis of the stability of slopes reinforced with piles against landslide in nonhomogeneous and anisotropic soils. Canadian Geotech. J. 45(8): 10921103

Shogaki T and Kumagai N 2008 A slope stability analysis considering undrained strength anisotropy of natural clay deposits. Soils Foundations 48(6): 805-819

Smith M 1993 Neural networks for statistical modeling. Thomson Learning

Su S and Liao H 1999 Effect of strength anisotropy on undrained slope stability in clay. Geotechnique 49(2): 215-230

Tsomokos A and Georgiannou V N 2010 Effect of grain shape and angularity on the undrained response of fine sands. Canadian Geotech. J. 47(5): 539-551. doi: 10.1139/T09-121

Uthayakumar M and Vaid Y 1998 Static liquefaction of sands under multiaxial loading. Canadian Geotech. J. 35(2): 273-283

Wright S G, Kulhawy F H and Duncan J M 1973 Accuracy of equilibrium slope stability analysis. J. Soil Mech. Foundations Div. 99(10): 783-791 